\title{
FIXED POINT THEOREMS IN UNIFORMLY CONVEX BANACH SPACES
}

\author{
MICHAEL EDELSTEIN ${ }^{1}$
}

\begin{abstract}
The notion of an asymptotic center is used to prove a number of results concerning the existence of fixed points under certain selfmappings of a closed and bounded convex subset of a uniformly convex Banach space.
\end{abstract}

1. Introduction. In this paper we shall assume that $X$ is a Banach space with positive modulus of convexity $\delta(\varepsilon)$ (i.e. $X$ is uniformly convex), where

$$
\begin{aligned}
\delta(\varepsilon)=\inf \left\{1-\frac{1}{2}\|x+y\|:\|x\| \leqq 1,\|y\| \leqq 1,\|x-y\|\right. & \geqq \varepsilon\} \\
& (0<\varepsilon \leqq 2) .
\end{aligned}
$$

Let $\left\{u_{n}: n=1,2, \cdots\right\}$ be a bounded sequence in a closed convex subset $C$ of $X$. As in [2] we define

$$
r_{m}(x)=\sup \left\{\left\|u_{n}-x\right\|: n \geqq m\right\}
$$

and denote by $c_{m}$ the unique point in $C$ with the property that

$$
r_{m}\left(c_{m}\right)=\inf \left\{r_{m}(x): x \in C\right\} .
$$

It was shown in [2] that a point $c$, called the asymptotic center of $\left\{u_{n}\right\}$ with respect to $C$, exists such that $c_{m} \rightarrow c$ as $m \rightarrow \infty$.

Some basic properties of the asymptotic center are collected in $\$ 2$ of the present paper. These are then used to obtain a much stronger version of a fixed point theorem proved first in [2]. Next, a fixed point theorem for a countable family of commuting mappings, more general than nonexpansive ones, is proved. A special feature of our results is that, given an orbit of an arbitrary point in $C$, the location of the fixed point is known a priori (as it coincides with the asymptotic center of that orbit) and it has certain uniqueness properties.

Received by the editors July 5, 1972.

AMS (MOS) subject classifications (1970). Primary 47H10, 46B99; Secondary $54 \mathrm{G} 60$.

Key words and phrases. Asymptotic center, fixed points, uniformly convex Banach space.

1 Visiting professor at the University of Adelaide; on sabbatical leave from Dalhousie University.

(c) American Mathematical Society 1974 


\section{Preliminaries.}

2.1. We shall make use of the mapping $r$ of $X$ into the reals defined by

$$
r(x)=\inf \left\{r_{m}(x): m=1,2, \cdots\right\} .
$$

The mapping $r$ as well as the $r_{m}(m=1,2, \cdots)$ are continuous on all of $X$. This follows easily from the fact that $r_{m}(x) \rightarrow r(x)$ as $m \rightarrow \infty$ and

$$
\left|r_{m}(x)-r_{m}\left(x^{\prime}\right)\right| \leqq\left\|x-x^{\prime}\right\| \quad\left(x, x^{\prime} \in X: m=1,2, \cdots\right) .
$$

Also, $r_{m}\left(y_{m}\right) \rightarrow r(y)$ as $y_{m} \rightarrow y$; for

$$
\begin{aligned}
\left|r_{m}\left(y_{m}\right)-r(y)\right| & \leqq\left|\left(r_{m}\left(y_{m}\right)-r_{m}(y)\right)+\left(r_{m}(y)-r(y)\right)\right| \\
& \leqq\left\|y_{m}-y\right\|+\left|r_{m}(y)-r(y)\right| .
\end{aligned}
$$

2.2. For the asymptotic center $c$ of $\left\{u_{n}\right\}$ with respect to $C$ we have

$$
x \in C \sim\{c\} \Rightarrow r((x+c) / 2)<r(x) .
$$

Indeed, since $c_{n} \rightarrow c$ [2, Theorem 1] there is an $N$ such that, for $n \geqq N,\left\|c_{n}-x\right\|>\frac{1}{2}\|c-x\|$. By uniform convexity and the definition of $r_{n}$ and $c_{n}$ we thus have for $k \geqq n \geqq N$

$$
\left\|u_{k}-\left(x+c_{n}\right) / 2\right\| \leqq r_{n}(x)(1-\delta(\|x-c\| / 2 \rho))
$$

where $\rho$ is a positive constant (e.g. $\left.\rho=r_{1}(x)+1\right)$. Thus

$$
r_{n}\left(\left(x+c_{n}\right) / 2\right) \leqq r_{n}(x)(1-\delta(\|x-c\| / 2 \rho))
$$

and therefore

$$
r((x+c) / 2) \leqq r(x)(1-\delta(\|x-c\| / 2 \rho))<r(x)
$$

as claimed.

2.3. From (5) we conclude easily that

$$
x \in C \sim\{c\} \Rightarrow r(c)<r(x) .
$$

For, clearly, $r_{m}\left(c_{m}\right) \leqq r_{m}(y)$. Thus $r(c) \leqq r(y)$ for all $y \in C$. If, however, contrary to $(6), r(c)=r(x)$ then with $y=(x+c) / 2$ we would have, by (5), $r(c)>r(y)$.

2.4. If for some $n_{0}, n \geqq n_{0} \Rightarrow\left\|u_{n}-z\right\| \leqq\left\|u_{n}-c\right\|$ then $z=c$. Indeed if $m \geqq n_{0}$ the above implies that $r_{m}(z) \leqq r_{m}(c)$ and, therefore, $r(z) \leqq r(c)$ which, by (6) is only possible if $z=c$.

2.5. In the special case when $X$ is a Hilbert space then $c \in \operatorname{cl} \operatorname{co}\left\{u_{n}\right\}$, the closed convex hull of $\left\{u_{n}\right\}$.

Proof. If $v \in X$ is not in cl $\operatorname{co}\left\{u_{n}\right\}$ then there is a nearest point $c^{\prime}$ to $v$ in $\mathrm{cl} \operatorname{co}\left\{u_{n}\right\}$. The hyperplane $\left\{x \in X:\left\langle x-c^{\prime}, c^{\prime}-v\right\rangle=0\right\}$ separates $v$ from $c l \operatorname{co}\left\{u_{n}\right\}$ and we may clearly assume that $\operatorname{Re}\left\langle u_{n}-c^{\prime}, c^{\prime}-v\right\rangle>0$ 
$(n=1,2, \cdots)$. Hence

$$
\begin{aligned}
\left\|u_{n}-v\right\|^{2} & =\left\|u_{n}-c^{\prime}\right\|^{2}+\left\|c^{\prime}-v\right\|^{2}+2 \operatorname{Re}\left\langle u_{n}-c^{\prime}, c^{\prime}-v\right\rangle \\
& >\left\|u_{n}-c^{\prime}\right\|^{2} \quad(n=1,2, \cdots) .
\end{aligned}
$$

It follows from 2.4 above that $v$ cannot coincide with the asymptotic center $c$.

3. Fixed point theorems. A well-known theorem due to Browder [1], Göhde [3] and, in a somewhat stronger form, to Kirk [4] states that every nonexpansive mapping of a closed and bounded convex set, in a uniformly convex Banach space, into itself has a fixed point. The next theorem, while assuming substantially less on the mapping, proves the existence of a fixed point having a preassigned location.

THEOREM 1. Let $f: C \rightarrow C$ be a mapping of a closed convex subset $C$ of a uniformly convex Banach space into itself and let $\left\{f^{n}(x): n=1,2, \cdots\right\}$ be a bounded sequence of iterates of some $x \in C$ having the asymptotic center $c$ with respect to $C$. If an $N$ exists such that

$$
\left\|f^{n}(x)-f(c)\right\| \leqq\left\|f^{n-1}(x)-c\right\| \quad(n>N)
$$

then $f(c)=c$.

Proof. Let $c^{\prime}=f(c)$. Then

$$
\left\|f^{n}(x)-c^{\prime}\right\|=\left\|f^{n}(x)-f(c)\right\| \leqq\left\|f^{n-1}(x)-c\right\|, \quad n>N .
$$

Hence $r_{n}\left(c^{\prime}\right) \leqq r_{n-1}(c)(n>N)$ and, therefore, $r\left(c^{\prime}\right) \leqq r(c)$. By (6) this can only happen when $c=c^{\prime}$, i.e. $f(c)=c$.

3.1. Remarks. (1) The above theorem is stronger than Theorem 2 of [2] in that condition (7) requires less than its counterpart there which requires that the same inequality be satisfied not only for $c$ but for all points $v$ of some neighborhood $V$ of $c$.

(2) A mapping $f: C \rightarrow C$ has a fixed point if the following condition is satisfied:

For all $x, y \in C$ there is an $N=N(x, y)$ such that

$$
\left\|f^{n}(x)-f(y)\right\| \leqq\left\|f^{n-1}(x)-y\right\| \quad(n>N) .
$$

Indeed, given an arbitrary $x \in C$, choose $y=c$, the asymptotic center of $\left\{f^{n}(x)\right\}$. Then $f(c)=c$.

(Note that in the corresponding statement in [2] the misprint occurs involving the replacement of $f(y)$ and $y$ by $f^{n}(y)$ and $f^{n-1}(y)$ respectively.)

3.2. If (7) is replaced by

$$
\left\|f^{n}(x)-f^{\prime \prime}(c)\right\| \leqq\left\|f^{n-1}(x)-c\right\| \quad(n>N)
$$


where $m$ is a fixed positive integer then the argument used in the proof of Theorem 1 yields the fact that $f^{m}(c)=c$ i.e. $c$ is a periodic point. The proof of the following theorem is based in part on this observation.

THEOREM 2. Let $C$ and $f$ be as in Theorem 1 and suppose that for each $x \in C$ there is an $N=N(x)$ and $m=m(x)$ such that, whenever $c=c(x)$ is the asymptotic center of $\left\{f^{n}(x)\right\}$ and $c \notin\left\{f^{n}(x): n>N\right\}$,

$$
\left\|f^{n}(x)-f^{m}(c)\right\| \leqq\left\|f^{n-1}(x)-c\right\| \quad(n>N)
$$

with strict inequality in the case when $f^{n-1}(x) \neq c$.

Then, for each $x \in C, f(c)=c(=c(x))$.

Proof. As observed before, if $x \in C$ and $c=c(x), f^{m}(c)=c$. Suppose $y=c \neq f(c)$ so that $m>1$. Let $N^{\prime}=N(y), m^{\prime}=m(y)$ and $c^{\prime}=c(y)$. Since, again $f^{m^{\prime}}\left(c^{\prime}\right)=c^{\prime}$ we obtain from (10) $\left\|f^{n}(y)-c^{\prime}\right\|<\left\|f^{n-1}-c^{\prime}\right\|\left(n>N^{\prime}\right)$ showing that $\left\|f^{N^{\prime}+k}(y)-c^{\prime}\right\|$ decreases with increasing $k$. On the other hand

$$
\left\|f^{N^{\prime}}(y)-c^{\prime}\right\|=\left\|f^{N^{\prime}+m}(y)-c^{\prime}\right\|<\left\|f^{N^{\prime}+m-1}(y)-c^{\prime}\right\|
$$

which is impossible. Thus $c=f(c)$ proving the theorem.

3.3. In the case of nonexpansive mappings the asymptotic center $c$ of $\left\{f^{n}(x)\right\}$ for an arbitrary $x$ in $C$ is next shown to be the fixed point of $f$ which is closest to $\left\{f^{n}(x): n=0,1, \cdots\right\}$.

Proposition 2. Let $f: C \rightarrow C$ be a nonexpansive mapping of the closed and bounded convex set $C$ into itself. If $x \in C$ and $c$ is the asymptotic center of $\left\{f^{n}(x): n=0,1, \cdots\right\}$ then

$r(c)=\inf \left\{\left\|f^{n}(x)-c\right\|: n=0,1, \cdots\right\} \leqq \inf \left\{\left\|f^{n}(x)-\xi\right\|: n=0,1, \cdots\right\}$

for each fixed point $\xi$ of $f$.

Proof. From $f(\xi)=\xi$ it follows that $\left\|f^{n+1}(x)-\xi\right\| \leqq\left\|f^{n}(x)-\xi\right\|$ implying that $r_{n}(\xi)=\left\|f^{n}(x)-\xi\right\|$ and $r(\xi)=\inf \left\{\left\|f^{n}(x)-\xi\right\|: n=0,1, \cdots\right\}$. The conclusion now follows directly from (6).

4. Fixed points common to certain families of mappings. In this section we first prove the existence of a common fixed point to a sequence of commuting mappings satisfying conditions considerably weaker than nonexpansiveness. This is accomplished by producing a sequence of asymptotic centers $\left\{c^{(m)}\right\}$ and showing that its asymptotic center has the desired property. In Hilbert space such a fixed point has a nearest point property analogous to that stated in Proposition 1 for a single mapping. Finally Browder's theorem [1] on the existence of a common fixed point for a family of commuting nonexpansive mappings is shown to hold for the wider class satisfying condition (8). 
THEOREM 3. Let $C$ be a closed and bounded convex set in a uniformly convex Banach space and $\left\{f_{n}: n=0,1, \cdots\right\}$ a sequence of commuting mappings of $C$ into itself. Let $c^{(1)}$ be the asymptotic center of $\left\{f_{0}^{n}(x)\right\}$ for some $x=c^{(0)} \in C$ and, recursively, let $c^{(n)}$ be the asymptotic center of $\left\{f_{n-1}^{m}\left(c^{(n-1)}\right)\right\}$. Let $c$ be the asymptotic center of $\left\{c^{(n)}: n=0,1, \cdots\right\}$ and suppose that

and

$$
\begin{aligned}
& \left\|f_{k}\left(c^{(l)}\right)-f_{k}(c)\right\| \leqq\left\|c^{(l)}-c\right\| \quad\left(l \geqq l_{k}>k ; k=0,1, \cdots\right) \\
& \left\|f_{k}^{n}\left(c^{(l)}\right)-f_{k}\left(c^{(l+1)}\right)\right\| \leqq\left\|f_{k}^{n-1}\left(c^{(l)}\right)-c^{(l+1)}\right\| \\
& \quad(n \geqq N(l, k) ; l, k=0,1, \cdots)
\end{aligned}
$$

$$
\begin{aligned}
\left\|f_{k} f_{l-1}^{n}\left(c^{(l-1)}\right)-f_{k}\left(c^{(l)}\right)\right\| \leqq\left\|f_{l-1}^{n}\left(c^{(l-1)}\right)-c^{(l)}\right\| \\
\quad(n \geqq N(l, k) ; l, k=1,2, \cdots) .
\end{aligned}
$$

Then $f_{k}(c)=c$ for all $k=0,1, \cdots$.

Proof. It suffices to show that for all $l>k, k=0,1, \cdots$,

$$
f_{k}\left(c^{(l)}\right)=c^{(l)}
$$

Indeed, if this is true then by (11)

$$
\left\|c^{(l)}-f_{k}(c)\right\|=\left\|f_{k}\left(c^{(l)}\right)-f_{k}(c)\right\| \leqq\left\|c^{(l)}-c\right\|
$$

for $l \geqq l_{k}>k$ and $k=0,1, \cdots$. By 2.4 , then, $f_{k}(c)=c$. To prove (14) note that $f_{k}\left(c^{(k+1)}\right)=c^{(k+1)}$ by Theorem 1 as a consequence of (12). Assuming then that $f_{k}\left(c^{(l-1)}\right)=c^{(l-1)}$ for $l-1>k$ we get from (13)

$$
\begin{aligned}
\left\|f_{l-1}^{n}\left(c^{(l-1)}\right)-f_{k}\left(c^{(l)}\right)\right\| & =\left\|f_{k} f_{l-1}^{n}\left(c^{(l-1)}\right)-f_{k}\left(c^{(l)}\right)\right\| \\
& \leqq\left\|f_{l-1}^{n}\left(c^{(l-1)}\right)-c^{(l)}\right\| \quad(n \geqq N(l, k))
\end{aligned}
$$

implying, as before, that $f_{k}\left(c^{(l)}\right)=c^{(l)}$.

Proposition 3. Let $X$ be a Hilbert space and $\left\{f_{n}: n=0,1, \cdots\right\} a$ sequence of commuting nonexpansive mappings of a closed and bounded convex subset $C \subset X$ into itself. Let $\left\{c^{(n)}\right\}$ and $c$ be as in Theorem 3. If $\xi$ is any common fixed point of the $f_{n}$ 's then

$$
\inf \left\{\left\|c^{(n)}-c\right\|: n=0,1, \cdots\right\} \leqq \inf \left\{\left\|c^{(n)}-\xi\right\|: n=0,1, \cdots\right\} .
$$

Proof. It follows from 2.5 that $\left\|c^{(n+1)}-\xi\right\| \leqq\left\|c^{(n)}-\xi\right\|, n=0,1, \cdots$. For

$$
\left\|f_{n}^{k}\left(c^{(n)}\right)-f_{n}^{k}(\xi)\right\| \leqq\left\|c^{(n)}-\xi\right\| \quad(n=0,1, \cdots ; k=1,2, \cdots)
$$

and, therefore $c^{(n+1)}$, being in $\mathrm{cl} \operatorname{co}\left\{f_{n}^{k}\left(c^{(n)}\right): k=1,2, \cdots\right\}$, is in the closed 
ball $B\left(\xi,\left\|c^{(n)}-\xi\right\|\right)$. Hence the conclusion of the proposition follows in the same manner as that of Proposition 2.

THEOREM 4. Let $\left\{f_{a}: a \in A\right\}$ be a family of commuting continuous mappings of a closed and bounded convex set $C$ (in a uniformly convex Banach space $X$ ) into itself and suppose that for each $a \in A$ and $x, y \in C$ there is an $N(x, y ; a)$ such that for $n>N(x, y ; a)$

$$
\left\|f_{a}^{n+1}(x)-f_{a}(y)\right\| \leqq\left\|f_{a}^{n}(x)-y\right\| .
$$

Then there is a $\xi \in C$ such that $f_{a}(\xi)=\xi(a \in A)$.

Proof. Let $F_{a}$ be the set of fixed points of $f_{a}$. We then have to show that $\bigcap\left\{F_{a}: a \in A\right\} \neq \varnothing$. A standard, and often used, argument shows that the commuting property implies that the $F_{a}$ have the finite intersection property. Thus it suffices to show that each $F_{a}$ is weakly compact. In view of the reflexivity of $X$ it suffices then to show that each $F_{a}$ is a nonempty closed and convex subset of $C$. From 3.1(2) we know that $F_{a} \neq \varnothing \quad(a \in A)$. Let then $y, z \in F_{a}$ and $u=\lambda y+(1-\lambda) z$ with $0<\lambda<1$. Choose $n>\max (N(y, u ; a), N(z, u ; a))$. Then

$$
\left\|y-f_{a}(u)\right\|=\left\|f_{a}^{n+1}(y)-f_{a}(u)\right\| \leqq\left\|f_{a}^{n}(y)-u\right\|=\|y-u\|
$$

and, similarly, $\left\|z-f_{a}(u)\right\| \leqq\|z-u\|$. This, however, in any strictly convex Banach space is only possible when $f_{a}(u)=u$ i.e. $u \in F_{a}$. Closedness of each $F_{a}$ is obvious.

\section{REFERENCES}

1. F. E. Browder, Nonexpansive nonlinear operators in a Banach space, Proc. Nat. Acad. Sci. U.S.A. 54 (1965), 1041-1044. MR 32 \#4574.

2. M. Edelstein, The construction of an asymptotic center with a fixed-point property, Bull. Amer. Math. Soc. 78 (1972), 206-208. MR 45 \#1005.

3. D. Göhde, Zum Prinzip der kontraktiren Abbildung, Math. Nachr. 30 (1965), 251-258. MR 32 \#8129.

4. W. A. Kirk, A fixed point theorem for mappings which do not increase distance, Amer. Math. Monthly 72 (1965), 1004-1006. MR 32 \#6436.

Department of Pure Mathematics, University of Adelaide, Adelaide, Australia

Current address: Department of Mathematics, Dalhousie University, Halifax, Nova Scotia, Canada 